\title{
Efecto de un programa de Educación Física con actividades motrices para desarrollar el área motora en niños con discapacidad intelectual
}

\author{
Effect of a Physical Education program with motor activities to develop the \\ motor area in children with intellectual disabilities
}

\author{
'Carlos Ernesto Ogarrio Perkinson, ${ }^{2}$ Alejandrina Bautista Jacobo, \\ ${ }^{3}$ Nidia Carolina Barahona Herrejon, ${ }^{4}$ María Elena Chávez Valenzuela, \& ${ }^{5}$ Graciela Hoyos Ruiz
}

Ogarrio, C., Bautista, A., Nidia Carolina Barahona, N., María Elena Chávez, M. E., \& Hoyos, G. (2021). Efecto de un programa de Educación Física con actividades motrices para desarrollar el área motora en niños con discapacidad intelectual. Revista Ciencias de la Actividad Física UCM, 22(2), julio-diciembre, 32-43. http://doi.org/10.29035/rcaf.22.2.3

\section{RESUMEN}

El objetivo principal de esta investigación fue evaluar el efecto de un programa de educación física con actividades motrices en niños con discapacidad intelectual inscritos en el Centro de Atención Múltiple de la ciudad de Hermosillo, Sonora. Se realizó un estudio cuantitativo de diseño cuasi-experimental con medias repetidas pretest-postest no aleatorizado, siendo cada participante su propio control. La muestra por conveniencia estuvo conformada por 12 niños en un rango de edad de 7 a 10 años (9 \$1.04). La evaluación se llevó a cabo por medio del Inventario de Desarrollo Battelle, el cual se aplicó al inicio y al final del programa, el cual tuvo una duración de tres meses. Se utilizó material didáctico reciclado para estimular los aspectos motrices que se deseaban. Los resultados muestran cambios significativos en la coordinación corporal y en locomoción ( $p=0.006$ ). Se concluye que aplicar un programa de educación física con actividades motrices de manera regular, a niños con discapacidad, puede contribuir desarrollar de mejor manera las habilidades de motricidad superando su edad equivalente.

Palabras clave: Educación física; actividades motrices; discapacidad intelectual; coordinación corporal.

\section{ABSTRACT}

The main objective of this research was to evaluate the effect of a physical education program with motor activities in children with intellectual disabilities enrolled in the Multiple Attention Center of the city of Hermosillo, Sonora. A quantitative study of quasi-experimental design with repeated means pretest-posttest non-randomized, with each participant being his/her own control was carried out. The convenience sample consisted of 12 children in an age range of 7 to 10 years $(9 \pm 1.04)$. The evaluation was carried out by means of the Battelle Development Inventory, which was applied at the beginning and at the end of the three-month program. Recycled didactic material was used to stimulate the desired motor aspects. The results show significant changes in body coordination and locomotion ( $p=0.006)$. It is concluded that applying a physical education program with motor activities on a regular basis to children with disabilities can contribute to better develop motor skills beyond their age equivalent.

Key words: Physical education; motor activities; intellectual disability; body coordination.

\footnotetext{
${ }^{1}$ Universidad de Sonora. Hermosillo, Sonora, México. http://orcid.org/0000-0001-6158-6525 | ogarrio63@yahoo.com

2 Universidad de Sonora. Hermosillo, Sonora, México. http://orcid.org/0000-0001-8018-7546 | alejandrina.bautista@unison.mx

${ }^{3}$ Universidad de Sonora. Hermosillo, Sonora, México. https://orcid.org/0000-0002-9276-6278| nidiabara67@outlook.com

${ }^{4}$ Universidad de Sonora. Hermosillo, Sonora, México. http://orcid.org/0000-0001-9418-1321 | malenamanzana@gmail.com

${ }^{5}$ Universidad de Sonora. Hermosillo, Sonora, México. http://orcid.org/0000-0001-6134-3179| graciela.hoyos@unison.mx
} 
Ogarrio, C., Bautista, A., Nidia Carolina Barahona, N., María Elena Chávez, M. E., \& Hoyos, G. (2021). Efecto de un programa de Educación Física con actividades motrices para desarrollar el área motora en niños con discapacidad intelectual. Revista Ciencias de la Actividad Física UCM, 22(2), julio-diciembre, 32-43. http://doi.org/10.29035/rcaf.22.2.3

\section{INTRODUCCIÓN}

Según datos del Instituto Nacional de Estadística y Geografía en el censo del 2014, existe en la población mexicana cerca de 7.1 millones de personas (6\%) que manifiestan tener algún tipo de discapacidad (INEGI, 2017). En Sonora, esta cifra es de 146.437 personas (INEGI, 2017). Según la OMS, en el mundo existe una población entre el 3 y $7 \%$ con discapacidad intelectual (OMS, 2011). Es de suma importancia estimular y desarrollar las habilidades motrices en los niños con Discapacidad Intelectual (DI), ya que sus conexiones cerebrales tendrán un mayor avance mediante el estímulo traducido en movimiento. La conducta humana es uno de los últimos aspectos por consolidarse; desde la infancia el niño construye su propia personalidad y la educación es el medio social más adecuado para ello. Es necesario, por lo tanto, sistematizar los procesos de enseñanza y aprendizaje que rigen su conducta motriz; la educación física en la educación básica contribuye a tal fin.

Cuando nos encontramos con un niño que presenta necesidades educativas especiales, la valoración de su desarrollo psicomotor debe ser más profunda para localizar cuáles son los puntos fuertes y débiles que presenta, con el fin de elaborar un programa dirigido a un camino en el que pueda desenvolverse con la mayor independencia posible, según sea el caso. Existen pocos instrumentos de evaluación del desarrollo psicomotor para niños que presentan algún tipo de discapacidad o que cuenten con adaptaciones para estos niños, pues hemos evidenciado que en la mayoría de los Centros Especiales se utilizan guías para niños regulares, obteniendo así resultados subjetivos. El Inventario de Desarrollo de Battelle es uno de los test más utilizados y aceptados en Latinoamérica, presenta adaptaciones generales y, a su vez, evalúa cinco áreas del desarrollo y subáreas correspondientes como son: área personal/social, adaptativa, motora, de comunicación y cognitiva, englobando así todos los aspectos del niño, además es un instrumento de evaluación a corto y largo plazo. Los resultados a corto plazo pueden servir de base para actuaciones terapéuticas inmediatas, mientras que los resultados a largo plazo constituyen una medida del progreso del niño a lo largo del tiempo.

La Educación Física es una forma de intervención pedagógica que se extiende como práctica social; su objeto de estudio no es sólo el cuerpo del niño en su aspecto físico, sino su corporeidad, es decir, sus experiencias motrices, vivencias, deseos, motivaciones, aficiones y sus praxias. Esto implica, por lo tanto, organizar la estructura de la enseñanza a partir de competencias educativas y para la vida; que hagan significativo lo aprendido y pueda ser utilizado a través de sus respuestas motrices y formas de convivencia basadas en el respeto, la equidad de género, la inclusión y, sobre todo, en la comprensión del interculturalismo de un país diverso como el nuestro, México. Existen muchos estudios que reportan los beneficios de la práctica de la actividad física en la población en general (Barroso et al., 2016). En el caso particular de niños con discapacidad intelectual, estos beneficios son relevantes, puesto que gran parte de su independencia funcional depende de su condición física (Vallbona, 2003). Pese a eso, no existen muchas investigaciones en esta línea.

De acuerdo con Schalock (2009), la intervención en la DI debe ser con base a programas que respondan a las necesidades de las personas en áreas educativas, de la vida personal, laboral y social, en especial en habilidades adaptativas conceptuales, prácticas y sociales, por ser consideradas la base para alcanzar el máximo grado de autonomía e integración en contextos inmediatos, dado que implican competencia motora, cognitiva, personal y social.

Según Cerda (1990), para lograr un diagnóstico real se debe realizar un examen psicomotor donde se involucren los aspectos motrices básicos como las pruebas de habilidad manual, control de la postura y el equilibrio estático, el equilibrio dinámico, las coordinaciones, sincinesias, lateralidad, tono y relajación, escritura y grafismo, estructuración 
Ogarrio, C., Bautista, A., Nidia Carolina Barahona, N., María Elena Chávez, M. E., \& Hoyos, G. (2021). Efecto de un programa de Educación Física con actividades motrices para desarrollar el área motora en niños con discapacidad intelectual. Revista Ciencias de la Actividad Física UCM, 22 (2), julio-diciembre, 32-43. http://doi.org/10.29035/rcaf.22.2.3

espacio-temporal, imitación de gestos sin significado.

No se puede concebir una educación integral y más aún con niños que requieren fundamentalmente de actividades físicas para estimular sus habilidades motrices. Las necesidades actuales exigen una integración a la sociedad del sector con DI, los cuales pueden integrarse a la vida productiva y ser de cierta manera independientes y autosuficientes, por ello se deben facilitar las herramientas básicas para el mejor desarrollo de este grupo humano en situación vulnerable, estimulando sus habilidades por medio de la actividad física, no sin antes haber llevado a cabo un diagnóstico sobre las capacidades de los niños.

Este estudio se realizó en el Centro de Atención Múltiple (CAM) de la ciudad de Hermosillo, Sonora, en donde, como primera etapa se llevó a cabo un diagnóstico con la finalidad de conocer la situación en la que se encontraban los niños de esta institución, observando que son atendidos por dos educadoras especializadas en educación especial, al igual que no contaban con un profesor de educación física y de psicomotrista, agregando que no disponían de un aula para educación psicomotriz. Nuestro diagnóstico en primera instancia es la carencia de esa estimulación y por ello se requiere de una evaluación para conocer qué aspectos en el área motora tiene mayor retraso para poder estimularlas y mejorarlas.

El desarrollo del niño es de vital importancia y sobre todo en aquellos que tienen una condición discapacitante, como en este caso lo es el retraso mental. Este colectivo debe ser estimulado en mayor proporción para poder ser nivelado a su edad biológica o lo mejor posible; en nuestro campo profesional hemos estudiado y comprendido que el ser humano desarrolla su inteligencia a través del movimiento, de esa manera se comunica con el mundo que le rodea, a través de los sentidos y de su corporeidad.

El objetivo principal de esta investigación fue aplicar un programa de educación física con actividades motrices con la finalidad de observar los cambios en el desarrollo motor de niños con discapacidad intelectual, por tanto, se planteó la siguiente hipótesis de investigación:

El programa de educación física provoca un cambio significativo en el área motor del niño.

\section{METODOLOGÍA}

Se llevó a cabo un estudio cuantitativo de diseño cuasi-experimental con medias repetidas pretestpostest no aleatorizado, siendo cada participante su propio control. La muestra por conveniencia estuvo conformada por 12 niños con Discapacidad Intelectual del Centro de Atención Múltiple (CAM) de la ciudad de Hermosillo, Sonora, México. El 50\% de los participantes fueron del sexo femenino y el otro $50 \%$ del masculino. El rango de edad fue de siete a 10 años (Media $=9, \mathrm{DE}=1.04$ ).

A los participantes se les administro un programa de educación física con actividades motrices para estimular y desarrollar su habilidad motora; este tuvo una duración de tres meses, estructurado en 36 sesiones de 45 minutos, tres veces por semana, aplicado durante las clases regulares.

Los criterios de inclusión fueron:

a) Niños o niñas con Dl.

b) Niños o niñas que estén inscritos en el CAM de Hermosillo, Sonora.

c) Niños o niñas cuyos padres hayan firmado el consentimiento de participación voluntaria.

Criterios de exclusión:

a) Niños y niñas que no asisten con regularidad al CAM

b) Niños y niñas que no asistieron a las evaluaciones.

c) Niños y niñas que no cuenten con el consentimiento de sus padres.

Los niños/as fueron evaluados en el área motora antes y después de la administración del 
Ogarrio, C., Bautista, A., Nidia Carolina Barahona, N., María Elena Chávez, M. E., \& Hoyos, G. (2021). Efecto de un programa de Educación Física con actividades motrices para desarrollar el área motora en niños con discapacidad intelectual. Revista Ciencias de la Actividad Física UCM, 22 (2), julio-diciembre, 32-43. http://doi.org/10.29035/rcaf.22.2.3

programa de educación física por medio del Inventario de Desarrollo Battelle.

\section{Variables}

Dependientes: Área motora, evaluado través de los siguientes indicadores: Coordinación corporal, locomoción, motricidad fina y motricidad perceptiva.

Independientes: Programa de educación física

\section{INSTRUMENTO}

El instrumento utilizado fue el Inventario de Desarrollo Battelle de Newborg et al. (2004), el cual mide las habilidades del desarrollo en niños con edades comprendidas entre el nacimiento y los ocho años y se aplica de forma individual. El instrumento también se aplica para niños que presenten necesidades especiales. Está integrado por un total de 341 ítems agrupados en las siguientes áreas: Personal/social, Adaptativa, Motora, Comunicación y Cognitiva. Dentro de cada una de estas áreas, se encuentran un grupo de ítems que forman a su vez subáreas. Para esta investigación, el Inventario de Desarrollo Battelle fue utilizado para evaluar únicamente al área Motora, la cual está formada por 82 ítems que evalúan la capacidad del niño/a para usar y controlar los músculos del cuerpo y se agrupan en cinco subáreas:

A) Control muscular. Evalúa el desarrollo motor grueso y la capacidad para establecer y mantener control sobre los músculos para sentarse, estar de pie, pasar objetos de una mano a otra o realizar otras actividades semejantes.

B) Coordinación corporal. Evalúa también aspectos del desarrollo motor grueso: la capacidad para utilizar su sistema muscular y para establecer un control y una coordinación corporal cada vez mayores (rodar en el suelo, dar patadas, lanzar, atrapar, etcétera).

C) Locomoción. Evalúa la capacidad para utilizar los sistemas de musculatura de forma integrada con el fin de trasladarse de un sitio a otro.

D) Motricidad fina. Evalúa el control y la coordinación de brazos y manos (atrapar y soltar objetos, ensartar, cortar y doblar papel, abrir y cerrar puertas, etcétera).

E) Motricidad perceptiva. Evalúa la capacidad para integrar la coordinación muscular y las habilidades perceptivas en actividades concretas como formar torres, copiar círculos, dibujar, escribir, etcétera.

\section{Criterios de puntuación}

Para valorar la realización del niño en cada ítem se tomaron como criterios los siguientes valores de acuerdo a Newborg et al. (2004):

2 puntos: El niño responde de acuerdo con el criterio establecido.

1 punto: El niño intenta realizar lo indicado en el ítem, pero no consigue alcanzar totalmente el criterio establecido.

O puntos: El niño no puede o no quiere intentar un ítem, o la respuesta es una aproximación extremadamente pobre a la conducta deseada

La puntuación directa de cada subárea se obtuvo sumando las puntuaciones de cada ítem. La puntuación en el área motora gruesa se obtuvo sumando la puntuación de control muscular, coordinación corporal y la puntuación en locomoción. La puntuación total en el área Motora fina se obtuvo sumando la puntuación obtenida en motricidad fina con motricidad perceptiva. Por último, se obtiene la puntuación total Motora, sumando la puntuación total en el área motora gruesa con la puntuación total del área Motora fina.

\section{PROCEDIMIENTO}

Se diseñó un programa de educación física con actividades particulares dirigidas al desarrollo del área motriz para ser aplicado a niños con discapacidad intelectual que asistían al Centro de 
Ogarrio, C., Bautista, A., Nidia Carolina Barahona, N., María Elena Chávez, M. E., \& Hoyos, G. (2021). Efecto de un programa de Educación Física con actividades motrices para desarrollar el área motora en niños con discapacidad intelectual. Revista Ciencias de la Actividad Física UCM, 22 (2), julio-diciembre, 32-43. http://doi.org/10.29035/rcaf.22.2.3

Atención Múltiple (CAM) en la ciudad de Hermosillo, Sonora. Se hizo una solicitud a la Secretaría de Educación y Cultura del estado de Sonora para realizar el estudio en dicha institución. Posterior a esto, se obtuvo una entrevista con la directora del plantel para exponer los objetivos de la investigación. La actividad fue realizada por los investigadores, los cuales pertenecen a la Subdirección del Deporte de la Universidad de Sonora y egresados del Programa de Capacitación y Nivelación Académica de la Maestría en Atención a Poblaciones Especiales de la Universidad Autónoma de Chihuahua

Después de plantear los objetivos del estudio y los procedimientos a realizar a la dirección del plantel, el equipo investigador entregó esta información a los padres de los niños del CAM, mediante entrevistas que además enfatizaron el carácter voluntario de la participación de sus hijos en las actividades. Al inicio del programa se registraron algunos datos personales como: edad, domicilio, estatura, peso, historial clínico, si asistían a algún tipo de terapias extraescolares, si practicaban algún deporte y datos familiares de los padres. Si el niño estaba medicado, información sobre los horarios de su medicación, además, se platicó con los padres sobre recomendaciones de la vestimenta, calzado e higiene de los niños. El programa se aplicó sólo a los niños cuyos padres firmaron la carta de consentimiento.

\section{ACTIVIDADES}

La escala nos permitió evaluar la locomoción en actividades como: caminar alternados brazos, a diferentes lados, adelante, atrás, si puede seguir una línea recta, sube y baja gradas; diferentes posiciones en actividades como mantenerse en cuclillas, rodillas, si cruza piernas al sentarse; el equilibrio sobre un pie alternándolos, con ojos cerrados, caminar a diferentes lados sobre una tabla; coordinación de piernas saltos a diferentes alturas, cuerda, 10 saltos seguidos, saltos adelante y atrás; coordinación de brazos lanzamiento, bote del balón, levanta objetos del suelo; coordinación de manos, corta papel con tijeras líneas recta, curva, si puede atornillar una tuerca; esquema corporal en sí mismo y en un compañero señalando partes del cuerpo.

\section{Materiales}

Se utilizó material reciclado de colores llamativos y de fácil manejo para los niños, como huellas de madera, muñecos de trapo, boliches, bastones y pinzas de ropa, entre otros, donde ellos reforzaban aspectos de la motricidad como esquema corporal, equilibrio coordinación gruesa y fina, etc.

Es importante mencionar que las sesiones aplicadas no fueron continuas por cuestiones del calendario escolar (vacaciones de Semana Santa, festejos del Día del Niño, etc.)

\section{Fases de la investigación:}

$7^{\circ}$ fase: Se solicitó la autorización de la Secretaría de Educación y Cultura del estado de Sonora, de los directores del plantel y padres de familia.

$2^{\circ}$ fase: Se tomaron medidas e información de los niños participantes, incluyendo la evaluación inicial del desarrollo motor por medio de Inventario de Desarrollo Battelle.

$3^{\circ}$ fase: Se aplicó el programa de Educación Física con actividades motrices.

$4^{\circ}$ fase: Se llevó a cabo la evaluación final del desarrollo motor de los niños por medio del Inventario de Desarrollo Battelle. Las evaluaciones se realizaron dentro del Centro de Atención múltiple.

$5^{\circ}$ fase: Se recolectaron los datos y se llevó a cabo el análisis de éstos.

\section{ANÁLISIS ESTADÍSTICO}

Los análisis estadísticos fueron realizados con el programa SPSS (Statical Package for the Social Sciences) versión 22. Se reportan puntajes medios, medianas y desviaciones estándar para cada una de las variables antes y después de la intervención. Para encontrar diferencias significativas entre los puntajes del Inventario de Desarrollo Battelle, antes 
Ogarrio, C., Bautista, A., Nidia Carolina Barahona, N., María Elena Chávez, M. E., \& Hoyos, G. (2021). Efecto de un programa de Educación Física con actividades motrices para desarrollar el área motora en niños con discapacidad intelectual. Revista Ciencias de la Actividad Física UCM, 22(2), julio-diciembre, 32-43. http://doi.org/10.29035/rcaf.22.2.3

y después del programa, se utilizó la prueba no paramétrica de Wilcoxon, dado el incumplimiento de normalidad comprobada por medio de la prueba de Shapiro-Wilk. El nivel de significancia que se utilizó fue de 0.05

\section{RESULTADOS}

En la Tabla 1, se muestran las puntuaciones directas obtenidas en el área motora para la primera y segunda evaluación, obtenida a través del Inventario de Desarrollo Battelle para el grupo de niños que participaron

Al inicio del programa, en la subárea de coordinación corporal, los niños obtuvieron una media de 38.33 (DS = 7.05) de puntuación directa. Después del programa, hubo un aumento significativo ( $p=0.006)$ de 5.35 puntos en su control corporal. En lo que se refiere a locomoción, también se observaron cambios significativos. Antes del programa de educación física, los niños obtuvieron un puntaje medio de 23.58 (DS = 2.87) al finalizar, el puntaje subió a 25.50 (DS = 1.97). Con respecto al desarrollo motor fino, aunque se observan puntajes más altos después del programa, éstos no fueron significativos. En general, el programa si logra cambios significativos ( $p=0.009$ ) en el puntaje total del Inventario de Desarrollo Battelle, siendo más manifiestos estos cambios en motricidad gruesa.

Tabla 1

Valores medios, medianas y desviaciones estándar de los puntajes directos en el área motora gruesa, fina y en el total del Battelle antes y después del programa de Educación Física.

\begin{tabular}{lccc}
\hline Variables & $\begin{array}{c}\text { Media } \pm \text { DS, mediana } \\
\text { Antes }\end{array}$ & $\begin{array}{c}\text { Media } \pm \text { DS, mediana } \\
\text { Después }\end{array}$ & $P^{*}$ \\
\hline Coordinación corporal & $38.83 \pm 7.05,43$ & $44.183 \pm 5.30,45$ & 0.006 \\
\hline Locomoción & $23.58 \pm 2.87,24.5$ & $25.50 \pm 1.97,26$ & 0.006 \\
\hline Puntuación total motora gruesa & $62.42 \pm 9.50,68.5$ & $70.33 \pm 6.12,71.5$ & 0.001 \\
\hline Motricidad fina & $31.42 \pm 3.70,31.5$ & $32.33 \pm 4.73,34$ & 0.526 \\
\hline Motricidad perceptiva & $19.92 \pm 3.80,20.5$ & $21 \pm 5.20,19.5$ & 0.336 \\
\hline Puntuación total motora fina & $51.33 \pm 6.71,51.5$ & $53.33 \pm 8.24,55$ & 0.314 \\
\hline Puntuación Total Battelle & $113.75 \pm 13.08,118.5$ & $123.70 \pm 9.44,126.5$ & 0.009 \\
\hline
\end{tabular}

Nota: DS = desviación estándar. *p valor se obtuvo con la prueba de Wilcoxon a un nivel de significancia de 0.05 Fuente: elaboración propia.

\section{Descripción del desarrollo motor para el grupo de edad de 84 a 103 meses (7 a 8,5 años)}

En la Figura 1, se observan los puntajes medios del desarrollo motor antes y después del programa para los niños de 84 a 103 meses ( $N$ =3). En todas las áreas se observa un aumento en el puntaje de Battelle; este aumento se ve más marcado en lo que se refiere la coordinación corporal (9 puntos). Este grupo lo conforman un niñoy dos niñas, en que el avance fue más notorio entre las niñas. En locomoción se presentó un aumento de casi dos puntos. De igual forma, las niñas tuvieron un mejor desarrollo en esta área. En lo que respecta a las habilidades relacionadas con motricidad fina, se presentó un retraso significativo en el niño y en una de las niñas de este grupo, por lo que el aumento grupal fue solo de un punto y esto se debió al avance de la otra niña, quien tuvo un cambio significativo en esta área de 11 puntos. En motricidad perceptiva, las niñas tuvieron un aumento satisfactorio de tres puntos, sin embargo, el niño presentó un retraso de cuatro puntos. 


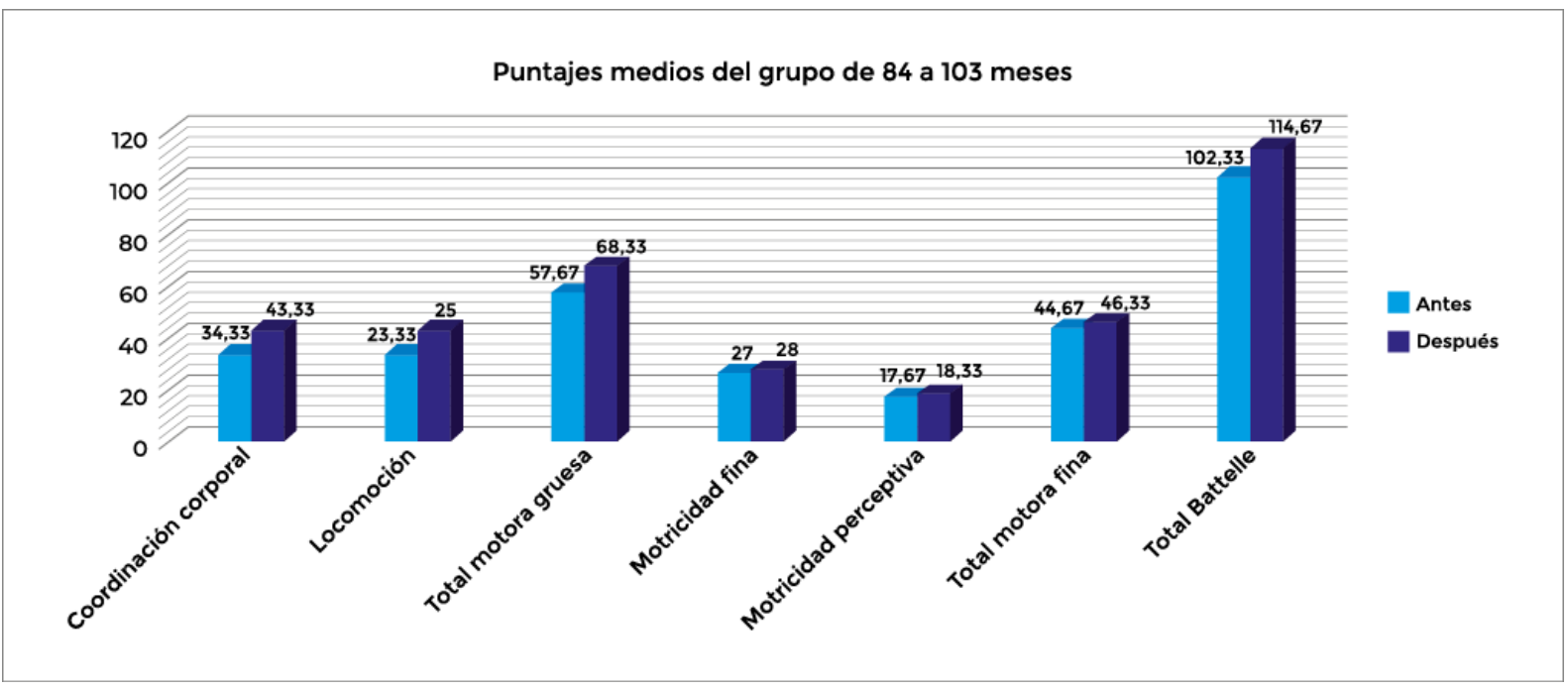

Figura 7. Puntajes medios en el área motora del grupo de 84 a 103 (7 a 8.5 años) meses antes y después del programa de Educación física. Fuente: elaboración propia.

\section{Descripción del desarrollo motor para el grupo de edad de 108 a 114 meses (9 a 9.5 años) antes y después del programa}

El grupo de 108 a 114 meses está constituido por cuatro niños y dos niñas. Al igual que en el grupo anterior, como se observa en la Figura 2, en el área de coordinación corporal y locomoción, las niñas obtuvieron un mayor cambio. En coordinación corporal hubo un aumento de casi cinco puntos, de manera que se puede informar que solo dos niños de este grupo tuvieron un retraso en las habilidades de coordinación, sobre todo para realizar saltos de longitud. El resto obtuvo en promedio un aumento de ocho puntos. En locomoción, se reporta un aumento grupal de tres puntos. En este grupo todos los niños obtuvieron un desarrollo en sus habilidades después del programa, a excepción de uno, que mantuvo su puntaje inicial. En lo que respecta a motricidad fina, de modo grupal se observa un cambio de dos puntos. De manera individual se menciona que el 50\% de los niños no hubo ningún efecto del programa, sin embargo, en el otro 50\%, uno de los niños reportó un aumento importante de ocho puntos en el desarrollo de sus habilidades, relacionado con el uso de las manos como cortar papel o ensartar cuencas. En motricidad perceptiva sucede algo similar, el grupo obtiene un aumento de un poco más de un punto, pero sólo el 50\% de los niños de este grupo logra un desarrollo satisfactorio de coordinación muscular, como copiar círculos o cuadrados. 
Ogarrio, C., Bautista, A., Nidia Carolina Barahona, N., María Elena Chávez, M. E., \& Hoyos, G. (2021). Efecto de un programa de Educación Física con actividades motrices para desarrollar el área motora en niños con discapacidad intelectual. Revista Ciencias de la Actividad Física UCM, 22(2), julio-diciembre, 32-43.

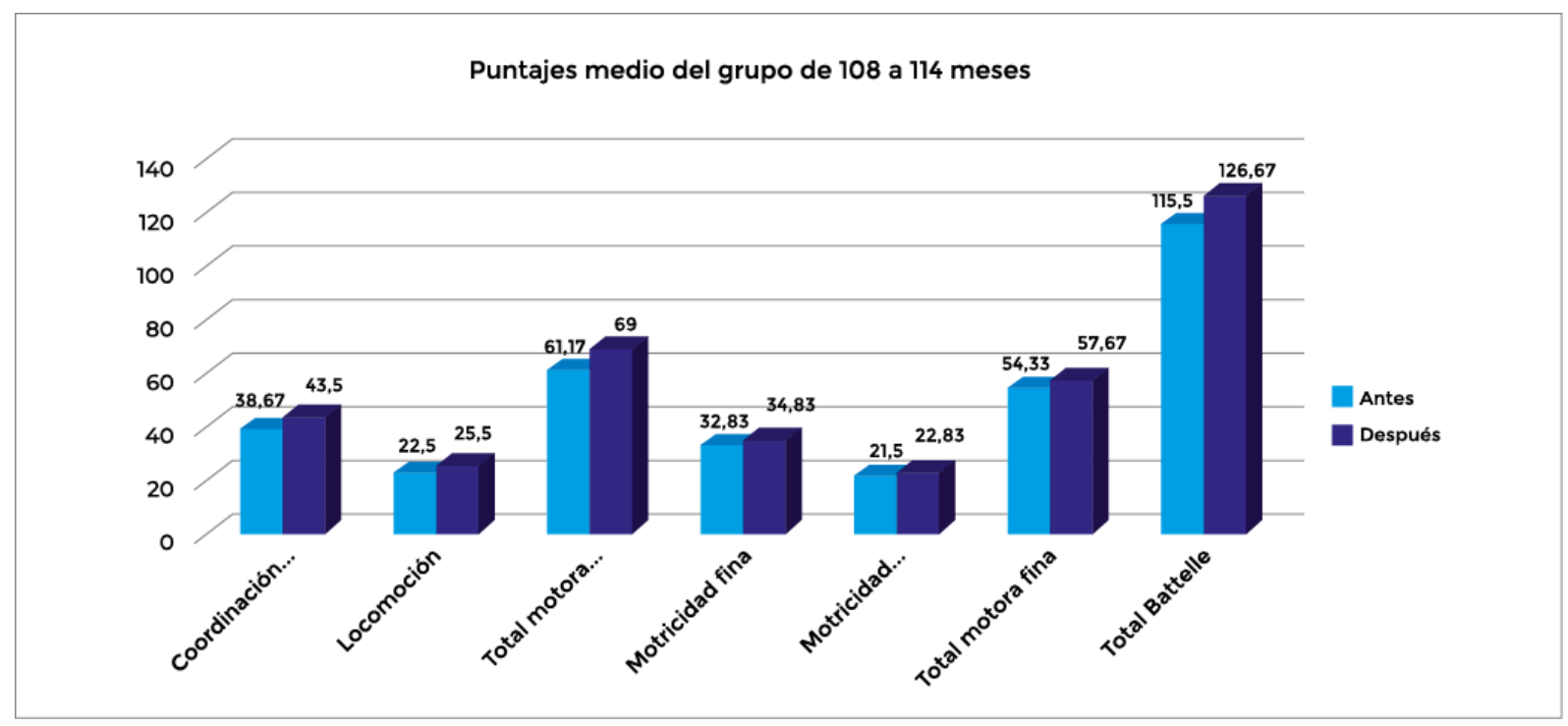

Figura 2. Puntajes medios en el área motora del grupo de 108 a 114 meses (9 a 9.5 años) antes y después del programa de Educación física. Fuente: elaboración propia.

Descripción del desarrollo motor para el grupo de edad de 119 a 123 meses antes y después del programa

El grupo 3, correspondiente a quienes cuentan con más de 9 años (19 a 123 meses), estuvo formado por sólo tres niños. En el área de coordinación corporal, los tres lograron un gran avance en las habilidades de motricidad gruesa, principalmente en actividades relacionadas con patear una pelota, lanzar o recoger objetos, sin embargo, en actividades como extraer algún objeto de una botella, solo uno logró aumentar su puntaje. En cuanto a las habilidades de motricidad fina $y$ perceptiva los resultados fueron similares, el programa causó un efecto positivo solo en uno de los niños (ver Figura 3).

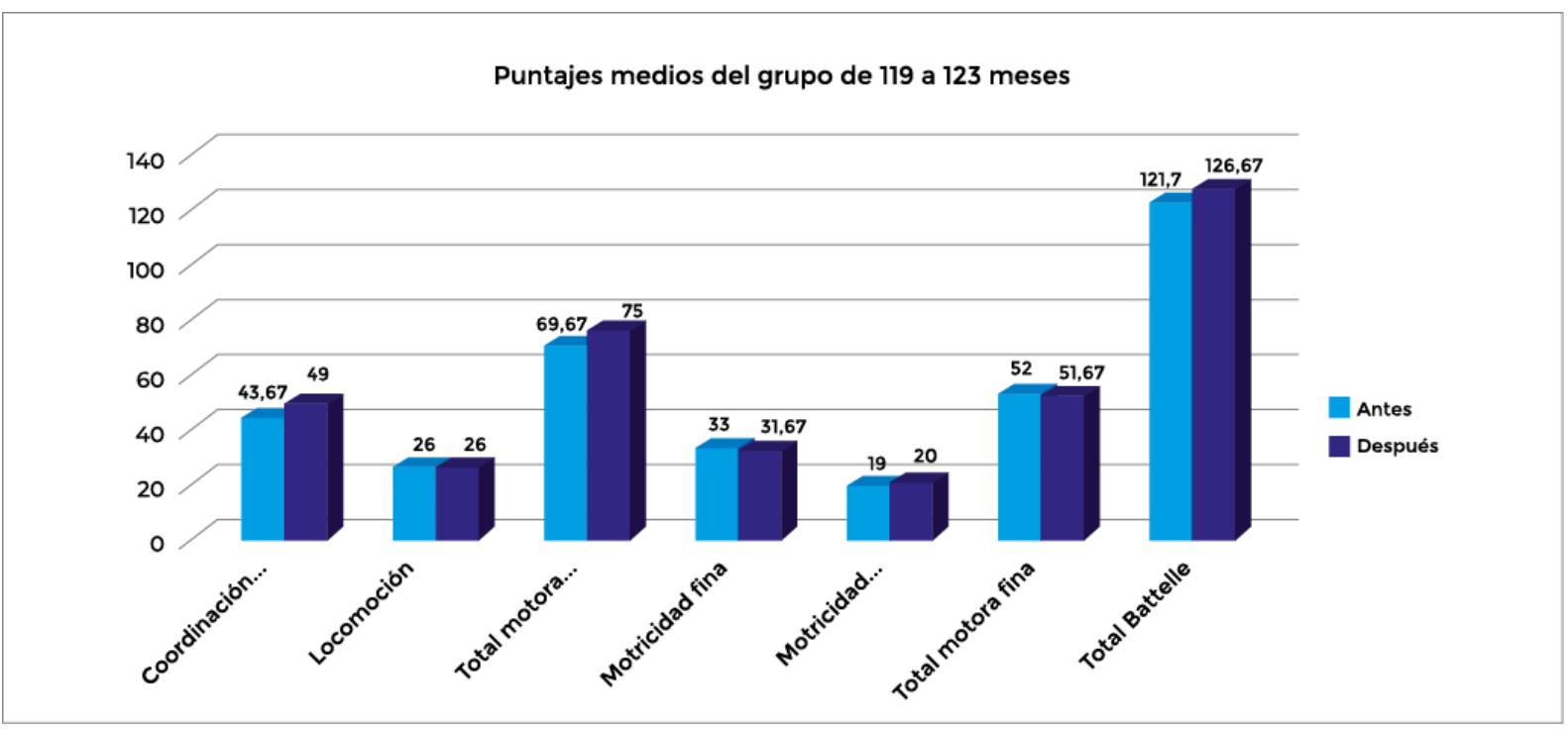

Figura 3. Puntajes medios en el área motora del grupo de 119 a 123 meses (más de 9 años) antes y después del programa de Educación física. Fuente: elaboración propia. 
Ogarrio, C., Bautista, A., Nidia Carolina Barahona, N., María Elena Chávez, M. E., \& Hoyos, G. (2021). Efecto de un programa de Educación Física con actividades motrices para desarrollar el área motora en niños con discapacidad intelectual. Revista Ciencias de la Actividad Física UCM, 22(2), julio-diciembre, 32-43. http://doi.org/10.29035/rcaf.22.2.3

Dados los puntajes de cada uno de los componentes del área motora, se determinaron las edades equivalentes en meses. En el área motor grueso, los niños antes del programa reportaban una edad equivalente de 41 semanas, al finalizar, el desarrollo de motricidad grueso fue equivalente a niños de casi 52 semanas ( $p=0.003$ ). En cuanto al área motor fino, los participantes tenían un desarrollo de niños de 48 semanas, después, su desarrollo sólo fue equivalente a niños de 50 semanas ( $p<0.05)$. En general, en el área motora, los niños reportaban una edad equivalente a 45 semanas. El programa logra aumentar su desarrollo equivalente a niños de 51 semanas ( $P=0.008)$. Ver Figura 4.

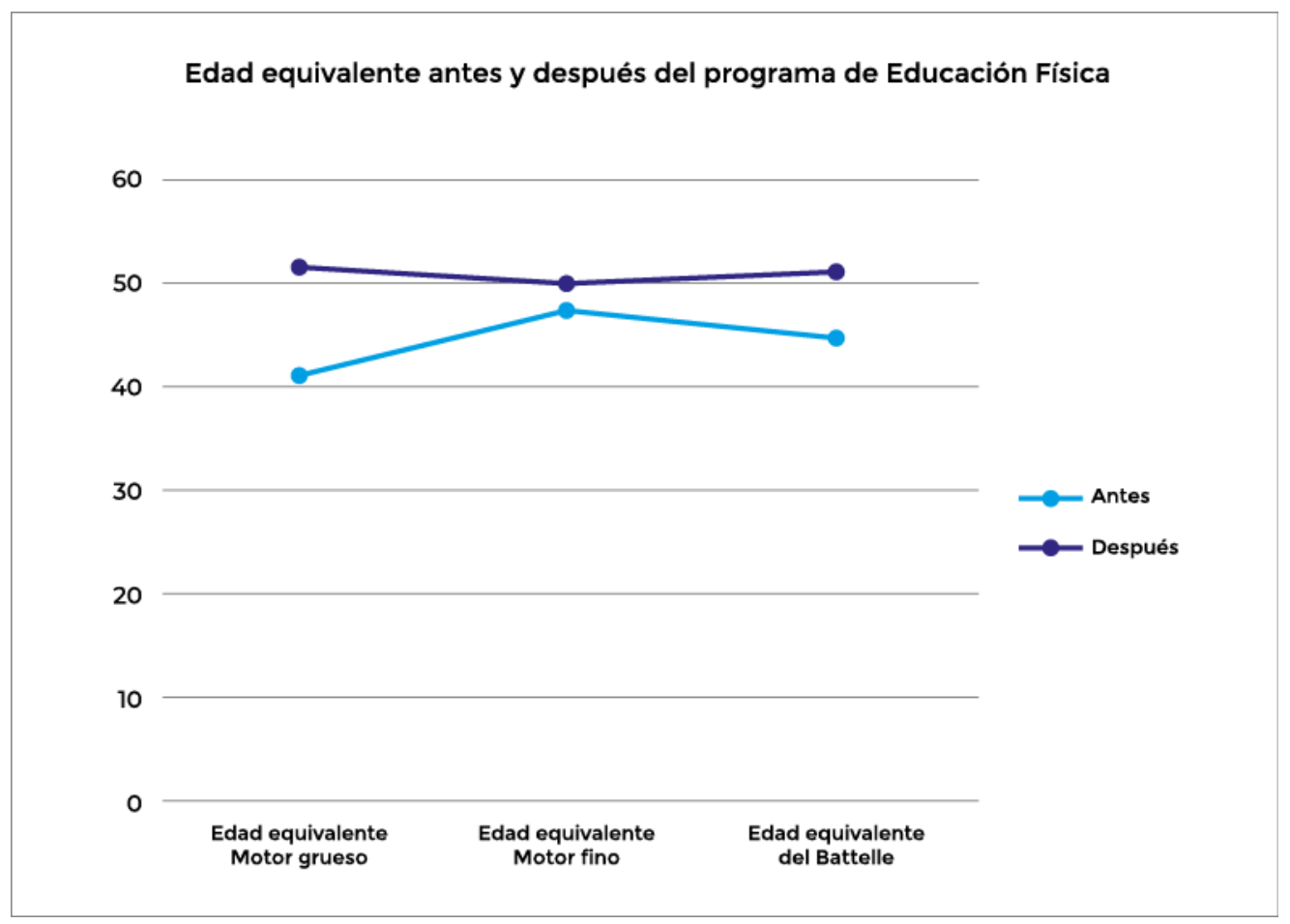

Figura 4. Edad equivalente antes y después del programa de Educación Física. Fuente: elaboración propia.

En general, el programa de Educación Física con actividades motrices logró un efecto positivo en el 83.3\% ( $n=10)$ de los niños en el área de coordinación corporal. En locomoción se logró un desarrollo en el 75\% ( $n=9$ ), un 16.7\% ( $n=2$ ) mantuvo igual el puntaje de Battelle y sólo un 8.33\% ( $n=1)$ presentó un retraso en las habilidades de esta área. Considerando el puntaje total del Inventario de Desarrollo Battelle, el programa logró un desarrollo satisfactorio en el 91.7\% de los niños en el área de motor gruesa.
En cuanto al área de motricidad fina, se logró un desarrollo satisfactorio en el 41.7\% ( $n=5)$ de los niños, un 25\% ( $n=3$ ) tuvo un retroceso y el 33.3\% ( $n=$ 4) mantuvo su puntaje original. En motricidad perceptiva, el programa causó un efecto positivo en el 50\% ( $n=6$ ) de los niños, el 25\% ( $n=3$ ) tuvo un retroceso y el otro $25 \%$ se mantuvo igual. En el puntaje total de la prueba de Battelle en el área motora fina, el programa logró un efecto positivo en el $41.7 \%$ ( $n=5)$ de los niños, un porcentaje igual tuvo un retroceso y el 16.7\% ( $n=2)$ se mantuvo igual. 
Ogarrio, C., Bautista, A., Nidia Carolina Barahona, N., María Elena Chávez, M. E., \& Hoyos, G. (2021). Efecto de un programa de Educación Física con actividades motrices para desarrollar el área motora en niños con discapacidad intelectual. Revista Ciencias de la Actividad Física UCM, 22(2), julio-diciembre, 32-43. http://doi.org/10.29035/rcaf.22.2.3

\section{DISCUSIÓN}

Los resultados de este estudio aportan evidencia sobre la importancia y el gran impacto que tiene un programa de Educación Física para desarrollar el área motora en niños con DI. El puntaje en coordinación corporal aumentó de manera significativa 5.35 puntos, evidenciando una mejora en la coordinación dinámica de los niños, que exige la capacidad de sincronizar los movimientos de diferentes partes del cuerpo como saltar, dar brincos y marometas. Así mismo, en locomoción, se logró un avance de casi dos puntos por arriba del puntaje inicial. Esto se observó en su capacidad para caminar, gatear y arrastrarse cuando se les entregaba la instrucción. Estudios en otras poblaciones han evidenciado los grandes beneficios no solamente físico, sino también mentales; tal es el caso del reportado por Sowa \& Meulenbroek (2012), quien implementó un programa de actividad física estructurada para niños y adultos con diferentes discapacidades, logrando aumentar de manera significativa las capacidades motoras y las actitudes sociales de los participantes. De igual forma, el estudio de Chasey \& Wyrick (1971), aplicaron un programa de desarrollo físico durante 15 semanas a una muestra de 27 niños. Las competencias motoras se midieron con las pruebas de Oseretsky. Los autores encontraron efectos significativos en las habilidades motoras gruesas en los niños. Resultados similares se han verificado en poblaciones de jóvenes con DI (Engel et al., 2018, Logan et al., 2018, Morgan et al., 2013). Mientras tanto, otros estudios se han enfocado en evaluar el efecto del ejercicio físico sobre los problemas de obesidad que suelen presentar algunos niños con alguna discapacidad, como es el síndrome de Down, evidenciando la influencia positiva no sólo sobre el área motora, sino también sobre la prevalencia de obesidad y sobrepeso en estas poblaciones (Dickinson \& Place, 2014, Fragala et al., 2008).

En cuanto al área motor fino, en este estudio no se obtuvo un avance significativo. Los niños no lograban coordinar bien los brazosy manos, de igual forma, presentaron dificultad para copiar círculos o dibujar y escribir. Se sabe que las actividades asociadas al área motor fino son de gran dificultad, según Durivage (1999), la motricidad fina consiste en la posibilidad de manipular los objetos, sea con toda la mano, sea con movimientos más diferenciados utilizando ciertos dedos. El niño adquiere la posibilidad de tomar una pinza alrededor de los nueve meses y la ejecuta con suma dificultad. Se requiere de una elaboración de años para realizar actividades motrices finas como enhebrar perlas y todavía más para llegar a la escritura, ya que ésta es una síntesis de las facultades neuro motrices y del desarrollo cognoscitivo.

Al comparar la evolución del antesy después en los grupos por edad, se encontraron cambios significativos en coordinación corporal en los tres grupos y sólo en dos en el área de locomoción. Tanto el área de motricidad fina como la perceptiva se observó un leve desarrollo en los dos primeros grupos por edad.

En lo que respecta a la edad equivalente, antes del programa, los niños desarrollaban actividades correspondientes a 47 semanas. El programa contribuyó particularmente al desarrollo las habilidades que vinculadas con el motor grueso, dado que ellos lograron desarrollar estas habilidades conforme a niños de 52 semanas. En las habilidades motoras finas no se logran grandes avances, pero tampoco hay un retroceso. Las actividades que desarrollaron después del programa fueron equivalentes a niños de 50 semanas, logrando lanzar pelotas, correr más de un metro sin caerse, pero no lograban extraer, por ejemplo, un objeto de una botella.

En general, los avances que se observaron de manera significativa corresponden al área motora gruesa, en donde casi el 100\% de los niños logró demostrar un gran progreso. Por otro lado, las áreas menos desarrolladas fueron las del motor fina. Esto debido a que los niños no comprendían las instrucciones o no podían traducirlas para ejecutar una acción concreta y por ello, se produjo una confusión en la información, al no tener una estructuración adecuada entre sus conexiones 
Ogarrio, C., Bautista, A., Nidia Carolina Barahona, N., María Elena Chávez, M. E., \& Hoyos, G. (2021). Efecto de un programa de Educación Física con actividades motrices para desarrollar el área motora en niños con discapacidad intelectual. Revista Ciencias de la Actividad Física UCM, 22(2), julio-diciembre, 32-43.

cerebrales y nerviosas, por lo que sus movimientos de desplazamiento eran torpes e inestables.

\section{CONCLUSIÓN}

Según las observaciones, se considera la necesidad de crear mecanismos que logren entablar una comunicación entre los niños con Dl y los maestros. Para los niños, la verbalización de palabras no se considera muy adecuada, más bien el comunicarse con el lenguaje corporal y no verbal se podría acercar más a su mundo, en vez de tratar de instruirlos desde nuestra perspectiva y conceptos sobre la docencia. Es importante procurar penetrar en su realidad, siendo ellos mismos quienes nos señalen las pautas a seguir. Los estudiosos de la naturaleza observan los fenómenos y luego entablan comunicación con ellos, por ejemplo, con los primates, equinos, delfines, canes, felinos.

La experiencia vivida en esta investigación sugiere que se deben adecuar nuevas metodologías para entablar la comunicación con niños con DI.

El programa tuvo una duración de tres meses, sin embargo, las actividades sociales y días asuetos interrumpieron el ritmo de trabajo. Se considera conveniente que este tipo de programa debe de ser parte fundamental en la enseñanza y formación, en este caso con niños con DI con una frecuencia de tres veces por semana y en coordinación con las otras actividades de expresión corporal y motricidad fina.

Aunque el tamaño de la muestra fue pequeño, los resultados combinados permiten llegar a la conclusión provisional de que, los niños con Dl que se someten a un programa de Educación Física con actividades motrices podrán desarrollar de mejor manera sus habilidades motoras y superar su edad equivalente.

\section{REFERENCIAS BIBLIOGRÁFICAS}

Barroso, G., Sánchez, B., \& Calero, S. (2016). Metodología para el desarrollo de programas de actividad física adaptada. Parte I: Estudio de la discapacidad en Ecuador. Lecturas: Educación Física y Deportes (EFDeportes.com), 20(212). https://www.efdeportes.com/efd212/estudi o-de-la-discapacidad-en-ecuador.htm

Cerda, C. (1990). Niños con necesidades educativas especiales. Promolibro.

Chasey, W., \& Wyrick W. (1971) Effects of a physical developmental program on psychomotor ability of retarded children. American Journal of Mental Deficiency, 75(5), 566-570. https://europepmc.org/article/med/5551501

Dickinson, K. \& Place, M. (2014). A randomised control trial of the impact of a computer-based activity programme upon the fitness of children with autism. Autism Reseach and Treatement, 2014, 1-9. https://doi.org/10.1155/2014/419653

Durivage, J. (1999). Educación y Psicomotricidad. Manual para el Nivel Preescolar. Trillas.

Engel, A., Broderick, C., Van Doorn, N., Hardy, L., \& Parmenter, B. (2018). Exploring the relationship between fundamental motor skill interventions and physical activity levels in children: A systematic review and meta-analysis. Sports Medicine, 48(8),18451857. https://doi.org/10.1007/s40279-0180923-3

Fragala-Pinkham, M., Haley, S., \& O’Neil, M. (2008). Group aquatic aerobic exercise for children with disabilities. Developmental Medicine and Child Neurology, 50(11), 822-827. https://doi.org/10.1111/j.1469 8749.2008.03086.x 
Ogarrio, C., Bautista, A., Nidia Carolina Barahona, N., María Elena Chávez, M. E., \& Hoyos, G. (2021). Efecto de un programa de Educación Física con actividades motrices para desarrollar el área motora en niños con discapacidad intelectual. Revista Ciencias de la Actividad Física UCM, 22(2), julio-diciembre, 32-43. http://doi.org/10.29035/rcaf.22.2.3

Instituto Nacional de Estadística y Geografía (INEGI). (2017). La discapacidad en México, datos al 2014: versión 2017. INEGI.

https://www.inegi.org.mx/contenidos/prod uctos/prod_serv/contenidos/espanol/bvine gi/productos/nueva_estruc/702825094409. pdf

Logan, S., Ross, S., Chee, K., Stodden, D. \& Robinson, L. (2018). Fundamental motor skills: A systematic review of terminology. Journal of sports Sciences, 36(7), 781-796. https://doi.org/10.1080/02640414.2017.13406 60

Morgan, P., Barnett, L., Cliff, D., Okely, A., Scott, H., Cohen, K., \& Lubans, D. (2013). Fundamental movement skill interventions in youth: $A$ systematic review and meta-analysis. Pediatrics, 132(5), el361-e1383. https://doi.org/10.1542/peds.2013-1167

Newborg, J., Stock, J., Wnek, L., De la Cruz, M., \& González, M. (2004). Battelle: Inventario de desarrollo. TEA
Organización Mundial de la Salud. (2011). Informe Mundial sobre la Discapacidad: Resumen. https://www.who.int/disabilities/world_rep ort/2011/summary_es.pdf

Schalock, R. (2009). La nueva definición de discapacidad intelectual, apoyos individuales y resultados personales. Siglo Cero, 40(229), 22-39. https://dialnet.unirioja.es/servlet/articulo?c odigo $=2985250$

Sowa, M., \& Meulenbroek, R. (2012) Effects of physical exercise on autism spectrum disorders: A meta-analysis. Research in Autism Spectrum Disorders, 6(1), 46-57. https://doi.org/10.1016/j.rasd.2011.09.001

Vallbona, C. (2003). La actividad física como elemento de salud para personas discapacitadas. En J. Martínez (Ed.), Conferencia Internacional sobre Deporte Adaptado. Libro de Actas (pp.283-296). Instituto Andaluz del Deporte.

Dirección para correspondencia

Carlos Ernesto Ogarrio Perkinson

Maestría en Atención a Poblaciones Especiales a Través del Movimiento

Facultad de Educación Física y Ciencias del Deporte

Universidad de Sonora.

Hermosillo, Sonora, México

Dirección postal: Agustín Melgar \#10, Colonia la Huerta, CP 83208 ,

Hermosillo, Sonora, México.

ORCID: http://orcid.org/0000-0001-6158-6525

Contacto:

ogarrio63@yahoo.com

Recibido: 10-12-2020

Aceptado: 09-06-2021 